\title{
Hotspot Advance Speed - Hotspot Size/Core-Hotspot Length Relation
}

\section{C.C Onuchukwu}

\author{
Department of Industrial Physics, Anambra State University Uli, Nigeria, PMB 02 Ihiala. \\ E-mail address: onuchukwu71chika@gmail.com
}

\begin{abstract}
Keywords: Galaxies General; Galaxies Active; Galaxies Jets; Methods Analytical; Methods Statistical; Methods Data Analysis
\end{abstract}

\begin{abstract}
We examined the evolution of extragalactic radio sources using the observed corehotspot length and hotspot size. Analysis indicates a fairly strong positive correlation in the ratio of core-hotspot length to hotspot size between that of the approaching arm and the receding arm with a correlation coefficient of $r \sim 0.7$. Simple kinematic consideration enabled us to obtain an expression to constrain the advance speed of hotspots. In general, the average projected hotspot advance speed estimated for our sample ranges from $0.13 \leq\langle\beta\rangle \leq 0.40$
\end{abstract}

\section{INTRODUCTION}

Active Galactic Nucleus (AGN) describes the nucleus of extragalactic radio sources (EGRS) with very high luminosities believed to be produced through accretion of matter onto a central object possibly a super massive black hole. The activity of the central engine is often accompanied by highly-relativistic collimated jets/beams (e.g. Begelman et al. 1984) of plasma material formed and accelerated in the vicinity of the black hole (Blandford \& Konigl 1979) which transport some of the accretion power to the lobes of EGRS where they are thermalized in the hotspots (Scheuer 1974). A long debated issue is the speed $(\beta)$ which these hotspots advance away from the AGN core, where $\beta$ is the bulk advance speed of the hotspot in units of $\mathrm{c}$ the speed of light.

Observational evidence of the relativistic jets in AGN include one-sided jet morphology, high brightness temperature of the AGN core determined with interferometers and from flux density variations (e.g. Kellermann 1968; Kellermann \& Pauliny-Toth 1981; Kellermann et al. 2004; Lähteenmäki \& Valtaoja 1999; Cohen et al. 2007), the asymmetry of the polarization at two jet sides (the so-called Laing-Garrington effect, Garrington et al. 1988; Laing 1988) and the observed apparent superluminal motion (e.g. Whitney 1971; Cohen et al. 1977; Jorstad et al. 2001; Kellermann et al 2004; Piner et al. 2007). In most high luminosity radio sources with one-sided kiloparsec scale jets, the observed parsec jets always points in the same direction of the kiloparsec jets (Pearson \& Readhead 1988; Wardle \& Aaron 1997), implying the persistence of relativistic motions in kiloparsec region of EGRS. Hardcastle et al. (1999) reported that relativistic beaming is needed to explain the observed relationship between core and jet prominence in their sample.

The advance speed of the hotspots/lobes for asymmetric radio sources can be estimated using the asymmetry parameters such as the arm-length ratio and apparent flux density ratio (e.g. Banhatti 1980, Best et al. 1995; Scheuer 1995, Ryś 2000), but this depends highly on the degree of asymmetry and he assumption that the observed asymmetry is entirely due to relativistic beaming. However, environmental and other intrinsic factors have been shown to be important in interpreting observed asymmetries in EGRS (e.g. McCarthy et al. 1991; Wardle \& Aaron 1997; Jeyakumar \& Saikia 2000; Jeyakumar et al. 2005; Onuchukwu \& Ubachukwu 2013). Another method for estimating hotspot advance speed is the synchrotron age estimates (Liu et al. 1992; Parma et al. 1999; Murgia et al. 1999; Schoenmakers et al. 2000; Jamrozy et al. 2005), but the uncertainty is large. The major source of uncertainty in estimating hotspot advance speed using synchrotron age estimates lies in the assumption of minimum-energy field in the estimation of the magnetic field $(B)$, since synchrotron age $\left(t_{s y n}\right)$ estimate depends more on magnetic field than the radio spectrum break frequency $v_{b}$ as $\left(t_{s y n} \propto B^{-1.5} v_{b}^{-0.5}\right.$ (see Longair 1981; De Young 2002), uncertainty in the value of magnetic field makes it difficult to derive the source age. 
In this article, we wish to estimate the projected advance speed of hotspot using the observed core-hotspot length and hotspot size. In this method we will assume constant advance speed of hotspot from the core and constant aspect ratio. The use of core-hotspot length rather than core-lobe length is due to the fact that hotspots are more compact and brighter thus angular extension should be more clearly defined. Gopal-Krisna \& Wiita (2004) noted 'that the main thrust of the collimated energy supply from the galactic nucleus is focused through the jets into the hotspots, any relativistic motion is more likely to be associated with them (Longair, Ryle \& Scheuer 1973; Blandford \& Rees 1974; Scheuer 1974), and not with the extended lobes'. In Section 2, we obtain an analytical expression that relates the hotspot expansion to hotspot size and core-hotspot length, in Section 3, we carry out the analysis and conclude in Section in section 4.

\section{THEORY OF RELATIONSHIP}

According to the standard beam model of radio-loud AGNs, it is believed that a fraction of accretion material forms an outflow (beam of relativistic plasma) along the rotational axis of the black hole (Blandford \& Znajek 1976; Mundell et al. 2003). These beams carry away the bulk kinetic energy from the central engine to kiloparsec regions $\sim 100-1000 k p c$ away from the central engines where they terminate and are thermalized at the hotspots (Blandford \& Rees 1974). Scheuer (1974) noted that the shocked relativistic plasma should expands sideways due to the interactions between the ambient intra cluster medium and the AGN jet. Assuming twin ejection of materials on the opposite sides of the central engine of AGN, and a simple kinematic model, in the frame of the source, the distance of the plasma element from the core $\left(D_{0}\right)$ can be described by

$D_{0}=\beta c t_{0}$

where $\beta$ is the bulk advance speed of the jets in units of $c$, the speed of light, $t_{0}$ is the time taken for the plasma element to traverse the distance $\left(D_{0}\right)$. Putting into consideration time delay effects and orientation effect, the observed time in the AGN rest frame is related to time in the observer's frame by, for the approaching arm (see Gopal-Krishna \& Wiita 2004)

$t_{a}=\gamma(1-\beta \cos \theta) t_{0}$

and for the receding arm,

$t_{a}=\gamma(1-\beta \cos \theta) t_{0}$,

where $\theta$ is the angle to the line of sight of a distant observer, $\gamma$ is the Lorentz factor related to the bulk speed by $\gamma=\frac{1}{\sqrt{1-\beta^{2}}}$. Thus, the time $(t)$ taken to traverse a given distance in the frame of the plasma element in terms of the observed distances, for the approaching arm $\left(D_{a}\right)$ can be written as

$$
t_{a}=\frac{D_{a}}{\beta c \sin \theta} \gamma(1-\beta \cos \theta)(1+z)
$$

and for the receding arm $D_{r}$ ) we have

$$
t_{r}=\frac{D_{r}}{\beta c \sin \theta} \gamma(1+\beta \cos \theta)(1+z)
$$

Here $D$ is the projected core-hotspot length, the subscripts $a$ and $r$ represents approaching/receding arm respectively. We have defined $D_{0}=D \sin \theta$ (see Ubachukwu 1998); the factor $(1+\mathrm{z})$ is needed to transform the observed time to the time in the frame of our galaxy (see Blandford \& Konigl 1979; Homan et al. 2009). Thus, the ratio of the time to reach an observed distance can be written as

$\frac{t_{r}}{t_{a}}=\frac{D_{r}(1+\beta \cos \theta)}{D_{a}(1-\beta \cos \theta)}$

When the plasma is thermalized, they expand sideways. Following Körding \& Falcke (2004), the expansion speed will depend on the speed of sound $\left(\beta_{s}\right)$ in the plasma. Now let $R_{a}$ be the observed size of the approaching side hotspot and $R_{r}$ the observed size of the receding side hotspot. The time $\left(t_{R}\right)$ to reach an observed size for the approaching arm may be written (assuming an isotropic medium) as

$$
t_{a R}=\frac{R_{a}}{\beta_{s}}
$$

and for the receding arm we have 


$$
t_{r R}=\frac{R_{r}}{\beta_{s}}
$$

Assuming that the time to reach an observed size $\left(t_{R}\right)$ is related to the time $(t)$ to reach an observed core-hotspot length (Kawakatu et al. 2008, had shown that there is a correlation between hotspot size and core-hotspot length), by $t_{R} \propto t^{y}$ with $y=1$ for simplicity (other values of $y$ are possible), then, from the above equations, we have

$$
\frac{D_{r} R_{a}}{D_{a} R_{r}}=\frac{(1+\beta \cos \theta)}{(1-\beta \cos \theta)}=\frac{1-\bar{\beta}}{1+\bar{\beta}}
$$

where $\bar{\beta}=\beta \cos \theta$ is the projected advance speed of the hotspot on the plane of the sky.

We note that the assumption for $y=1$, may not be entirely true for different regimes of corehotspot length and expansion mode due to different modes of interactions expected between the jet plasma and the ambient medium in the host galaxy (e.g. Saikia et al. 1995; O'Dea 1998; Dallacasa et al. 2002). For sources with core-hotspot length less than $1 \mathrm{kpc}$ and for those with core-hotspot length greater than $1 \mathrm{kpc}$, Kawakatu et al. (2008) obtained a different slope to the linear fit of the core-hotspot length hotspot size relation in logarithm. Furthermore, the power-law index for the evolution of the hotspot size may change at transition between interstellar medium and intergalactic medium (e.g. Jeyakumar \& Saikia 2000; Perucho \& Marti 2002). But for constant jet advance speed and constant aspect ratio usually assumed in self-similar model treatment of extragalactic radio source (Begelman \& Cioffi 1989; Loken et al. 1992; Cioffi \& Blondin 1992), the assumption that the time $\left(t_{R}\right)$ to reach a given hotspot size correlates with the time $(\mathrm{t})$ to reach a given core-hotspot length with $\mathrm{y}=1$ seems reasonable. Equation (8) can be inverted to give

$$
\bar{\beta}=\frac{1-b}{1+b}
$$

where $b=\frac{D_{r} R_{a}}{D_{a} R_{r}}$ and gives us an expression to constrain the projected advance speed of hotspots for sources with observed core-hotspot length and hotspot size. In general, a linear regression fit to equation (8) in the form

$$
\log \left(\frac{D_{r}}{R_{r}}\right)=\log \left(\frac{D_{a}}{R_{a}}\right)+\log \left(\frac{1-\bar{\beta}}{1+\bar{\beta}}\right)
$$

will enable us estimate the average projected bulk expansion speed $\bar{\beta}$. Also, the $\log \left(\frac{D_{r}}{R_{r}}\right)-\log \left(\frac{D_{a}}{R_{a}}\right)$ plot is theoretically expected to yield a slope of 1 . This can be tested using a well-defined source sample. The logarithmic form of the relationship was chosen due to the expected wide spread in the observed core-hotspot length and hotspot size.

\section{DATA ANALYSIS/RESULT}

The analyses were based on a sample of extragalactic radio sources obtained from Kawakatu et al. (2008) which they culled from literature. According to Kawakatu et al. (2008), to minimize the difference in estimation of physical quantities between approaching hotspot and counter hotspot, they selected mainly sources with relatively symmetric lobes. For our analysis, we selected sources with observed information on jet and counter jet core-hotspot length and hotspot size. We excluded 3 sources $(1005+070,0255+460$ and 1314+453) with core-hotspot length and hotspot size exactly the same for both arms, these sources will give a ratio of 1 , and thus not suitable for our expression in estimating projected hotspot advance speed. The final sample consists of 98 sources classified as Compact Symmetric Objects (CSO), Medium-size Symmetric Objects (MSO), Compact Steep Spectrum Sources (CSS) and FR II sources. In Kawakatu et al. (2008), there was no identification of the approaching arm and the receding arm. Assuming simple relativistic beaming scenario (e.g. Ryle \& Longair 1967; Gopal-Krishna \& Wiita 2004), the longer arm is assumed to be the approaching arm.

In relativistic beaming scenario and twin beam model of $A G N$, for plasma elements ejected from the core and observed at the same time, the receding blob will be at a shorter distance from the core and smaller in size than the approaching blob. Thus, we expect a positive correlation in the ratio of core-hotspot length to hotspot size between that of the approaching arm and receding arm. The correlation coefficient result is strong for our sample with $r \sim 0.7$ with $p=3.4 \times 10^{-7}$ 
(where $p$ is the probability of getting the given value of $r$ by chance. Using equation (9), the average projected hotspot advance speed estimated for our sample is $\langle\bar{\beta}\rangle=0.3 \pm 0.2$, with the distribution plot of the estimated projected speed for each source shown in figure 1. Figure 2 shows the $\log \left(\frac{D_{r}}{R_{r}}\right)-\log \left(\frac{D_{a}}{R_{a}}\right)$ plot.

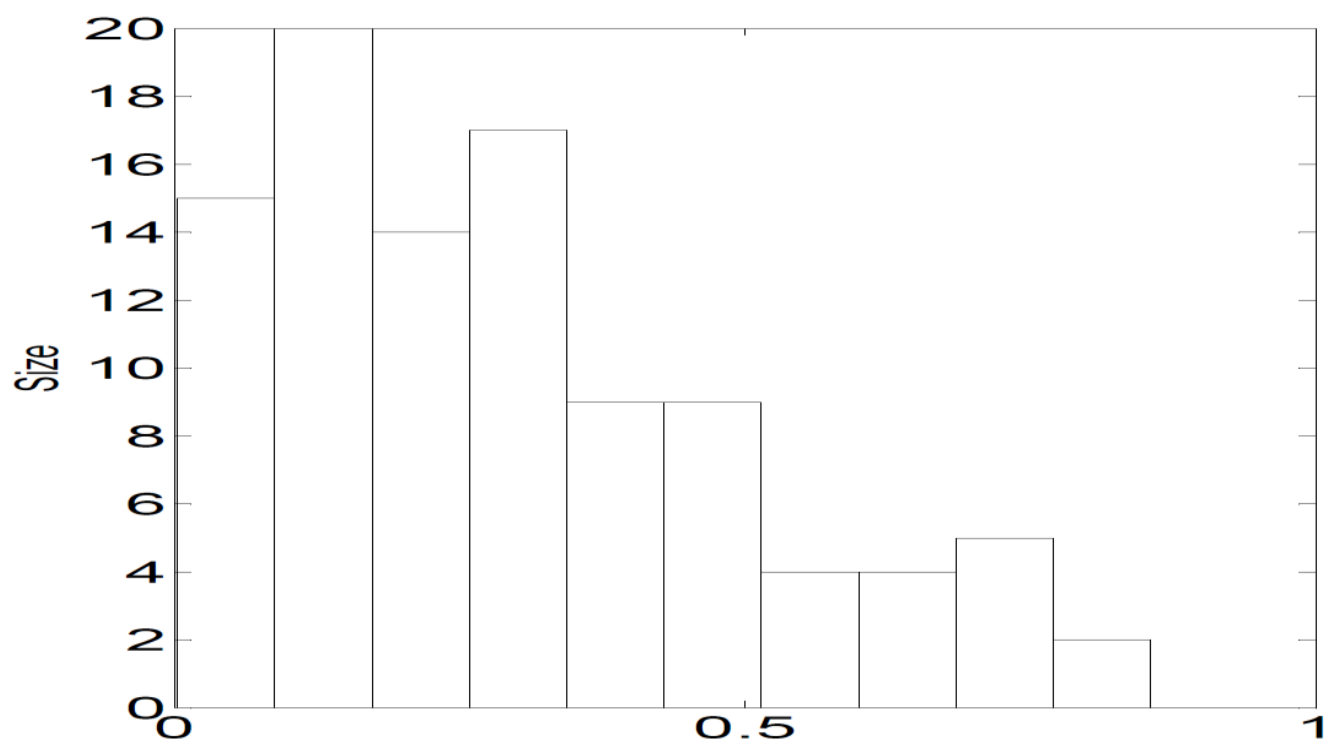

Fig 1 Histogram Plot of The Estimate Hotspot Advance Speed $(\beta \cos \theta)$ for Each Source in The Sample.

If the sources were sub-divided based on core-hotspot length (see Kawakatu et al. 2008) into CSO (with $D \leq 1 \mathrm{kpc}$ ), MSO (with $1 \leq D \leq 10 \mathrm{kpc}$ ) and FR II radio sources (with $D \geq$ $10 \mathrm{kpc}$ ), the average estimated projected hotspot advance speed is $\langle\bar{\beta}\rangle=0.3 \pm 0.2,0.4 \pm 0.1$ and $0.3 \pm 0.1$ for FR II, MSO and CSO sources respectively. A linear regression fit to the $\log \left(\frac{D_{r}}{R_{r}}\right)-$ $\log \left(\frac{D_{a}}{R_{a}}\right)$ plot (equation (11) gives: $\log \left(\frac{D_{r}}{R_{r}}\right)=(0.9 \pm 0.2) \log \left(\frac{D_{a}}{R_{a}}\right)-(0.11 \pm 0.03)$ this gives a mean projected advance speed of $\langle\bar{\beta}\rangle=0.13 \pm 0.03$. The slope of the regression fit to equation (11) agrees well with the theoretical value, indicating that our assumption is plausible.

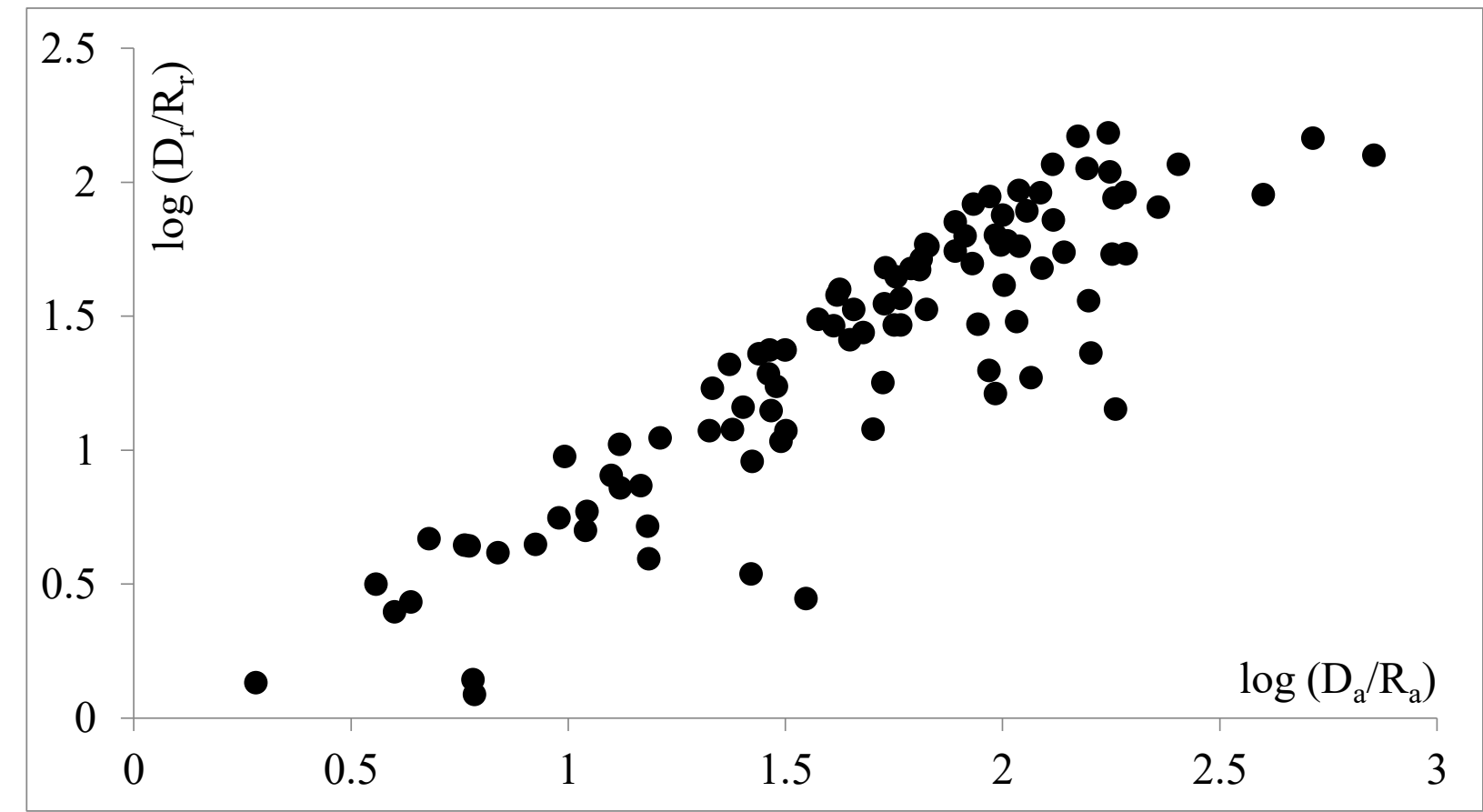

Fig 2. Plot of $\log \left(\frac{D_{r}}{R_{r}}\right)$ against $\log \left(\frac{D_{a}}{R_{a}}\right)$ For The Sources In Our Sample 


\section{DISCUSSION AND CONCLUSION}

We have investigated a simple consequence of kinematic evolution of hotspot size and core-hotspot length. We also used it to obtain an estimate for the projected hotspot advance speed of $0.1 \leq$ $\langle\bar{\beta}\rangle \leq 0.4$ for a sample of radio sources. There are several sources of uncertainties in the estimation of the projected hotspot advance speed using the observed hotspot size and core- hotspot length of radio sources. According to Kawakatu et al (2008), these include (i) hotspots sizes due to lack of sensitivity for diffused emission, the sizes of larger hotspot may be underestimated due to a lack of sensitivity for diffuse emission. (ii) Uncertainties in the angular resolution of the angular size of radio source may affect the estimation of the hotspot size. The higher the angular size resolution, the finer the details revealed and this might lead to underestimation of the hotspot sizes. (iii) Projection effect - Kawakatu et al. (2008) noted that the estimated size of the hotspot is not intrinsic size but the size projected onto the celestial plane, though the sample was restricted to the sources with relatively symmetric lobes.

The estimated speed within the limits of error, supports the assumption of constant advance speed of hotspots, though there seems to be a mild increase at the CSO $(\langle\bar{\beta}\rangle \sim 0.3)$, MSO $(\langle\bar{\beta}\rangle \sim 0.4)$ phase and deceleration at the MSO FR II $(\langle\bar{\beta}\rangle \sim 0.3)$ phase. The CSO - MSO phase seemly acceleration is expected for a power-law ambient density profile that scales approximately as King (1972) profile with $\rho_{D} \sim \rho_{0}\left(\frac{D}{D_{0}}\right)^{-\epsilon}$, where $\rho_{0}$ is the central density distribution of the source environment, and here $D_{0}$ is the assumed inner radius and $\epsilon$ index of decline. Thus, for $D \geq D_{0}$ there will be less interaction with environment and less obstruction to advance motion away from the core, leading to higher speeds. From the observed values of advance speed shown in Kawakatu et al. (2008), the average advance speed in CSO - MSO - FR II phases are $\beta \sim 0.13 ; 0.15$ and 0.05 respectively for CSOs, MSOs and FR IIs. These values follow similar trend (though of lower values) to the average projected advance speed we estimated. The assumption of simple relation between the time for hotspot growth and distance away from the core may have been responsible for the discrepancies in the two results. Our results may be considered the upper limit to the expansion speed while Kawakatu et al. (2008) result may reflect the lower limit.

Observations of the synchrotron radiation spectrum of extragalactic radio sources (assuming equipartition magnetic field) have led to the estimation of the advance speed of the hotspots for most powerful extragalactic radio sources as $\beta \sim 0.2-0.3$ (Myers \& Spangler 1985; Liu et al. 1992). Using the prevalence of long lobe of radio sources, Longair \& Riley (1979) estimated that the expansion speed of the lobes in general cannot be more than $\beta \sim 0.25$. Furthermore, Best et al. (1995) showed that a hotspot speed in the range $\beta \sim 0.2-0.3$ would be required to reproduce the arm-length ratio distribution for the 3CRR sample. More recently, Stanghellini et al. (2009) in their study of three compact radio sources obtained hotspot speed which lies in the range $\beta \sim 0.2-0.4$. Orienti \& Dallacasa (2010) reported a mean apparent expansion speed in intrinsically compact radio sources of $\beta \sim(039 \pm 0.18)$ which is in agreement with the values obtained by Polatidis \& Conway (2003) who studied a dozen of the most compact radio sources. These results are in general, consistent with the values obtained in this paper.

On the other hand, Scheuer (1995) obtained a somewhat lower value $\beta \leq 0.15$, with most probable speed value of $\beta \sim 0.03-0.02$ which may be taken as the lobe speed (a rapid backflow is expected if the jet material is much less dense than the ambient plasma); such strong backflows are clearly observed in many numerical simulations of jet propagation (see Norman 1996; Hooda \& Witt 1998). Furthermore, Arshakain \& Longair (2000) using the jet-sidedness ratio for a large sample of quasars and galaxies, obtained similar small values of hotspot advance speed of $\beta \sim 0.11 \pm 0.01$. However, in their analysis, they pointed out that intrinsic/and environmental effects also contribute to the observed asymmetries.

In conclusion, using the observed core-hotspot length, hotspot size and assumption of constant hotspot advance sped/aspect ratio, we obtained an expression that helped us constrained projected hotspot advance speed of extragalactic radio sources. 
Acknowledgements C.C Onuchukwu wishes to thank I. Ubah for his valuable discussions.

\section{References}

[1] Arshakain, T.G., \& Longair, M.S., 2000, MNRAS, 311, 846

[2] Banhatti, D. G., 1980, A\&A, 84, 112.

[3] Begelman, M. C., \& Cioffi, D. F. 1989, ApJ, 345, L21

[4] Begelman, M. C., Blandford, R. D., \& Rees, M. J. 1984, Rev. Mod. Phys., 56, 255

[5] Best, P.N., Bailer, M.D., Longair, M.S., \& Riley, J.M., 1995, MNRAS, 275, 1171

[6] Blandford R.D., \& Rees, M.J., 1974, MNRAS, 169, 395.

[7] Blandford, R.D., \& Znajek, R.L., 1976, MNRAS, 179, 433

[8] Blandford R.D., \& Konigl A., 1979, ApJ, 232, 34

[9] De Young, D.S., 2002, The Physics of Extragalactic Radio Sources, University of Chicago Press Limited. Chicago. pp557

[10] Cohen, M.H., Linfield, R.P., Moffet, A.T., Seielstad, G.A., Kellermann, K.I., Shaffer, D.B., Pauliny-Toth, I.I., Preuss, E., Witzel, A., \& Romney, J.D., 1977, Nature, 268, 405

[11] Cohen, M. H., Lister, M. L., Homan, D. C., Kadler, M., Kellermann, K. I., Kovalev, Y. Y., \& Vermeulen, R. C. 2007, ApJ, 658, 232

[12] Cioffi, D.F. \& Blondin, J.M., 1992, ApJ, 392, 458

[13] Dallacasa, D., Fanti, C., Giacintucci, S., Stanghellini, C., Fanti, R., Gregorini, L., \& Vigotti, M. 2002, A\&A, 389, 126

[14] Garrington, S.T., Leahy, J.P., Conway, R.G., \& Laing, R.A., 1988, Nature, 331, 147

[15] Gopal-Krishna \& Wiita, P. J., 2004, e-print arXiv:astro-ph/0409761

[16] Hardcastle, M.J., Alexander P., Pooley G.G., \& Riley J.M., 1999, MNRAS, 304, 135

[17] Homan, D.C., Kadler, M., Kellermann, K.I., Kovalev, Y.Y., Lister, M.I., Ros, E., Savolainen, T. \& Zensus, J.A., 2009, 706, 1253

[18] Hooda, J.S. \& Wiita, P.J., 1998, ApJ, 493, 81

[19] Jamrozy, M., Machalski, J., Mack, K.-H., \& Klein, U. 2005, A\&A, 433, 467

[20] Jeyakumar, S., Saikia, D. J. 2000, MNRAS, 311, 397J

[21] Jeyakumar, S., Wiita, P. J., Saikia, D. J., \& Hooda, J. S., 2005, A\&A, 432, 823J

[22] Jorstad S.G., Marscher, A. P., Mattox, J. R., Wehrle, A. E., Bloom, S. D., \& Yurchenko, A. V., 2001, ApJS, 134, 181

[23] Kawakatu, N., Nagai, H., \& Kino, M., 2008, ApJ, 687, 141

[24] Kellermann K.I., 1968, ARA\&A, 6, 417

[25] Kellermann K.I. \& Pauliny-Toth I.I.K. 1981, ARA\&A, 19, 373

[26] Kellermann, K. I., Lister, M. L., Homan, D. C., Vermeulen, R.C., Cohen, M. C., Ros, E., Kadler, M., Zensus, J.A., \& Kovalev, Y. Y., 2004, ApJ, 609, 539

[27] King, I.R., 1972, ApJ, 174, L123

[28] Körding, E., \& Falcke, H., 2004 in the role of VLBI in Astrophysics Astronomy and Geodesy eds. F. Montavani and A. Kus, Kluwer Academic Publishers, Netherlands, 107127 
[29] Laing, R.A., 1988, Nature, 331, 149

[30] Lähteenmäki, A., \& Valtaoja, E., 1999, ApJ, 521, 493

[31] Liu, R., Pooley, G.G., \& Riley, J.M., 1992, MNRAS, 257, 545

[32] Loken, C., Burns, J.O., Clarke, D.A. \& Norman, M.L., 1992, ApJ, 392, 54

[33] Longair, M.S., 1981, High Energy Astrophysics, Cambridge University Press. Cambridge. pp412

[34] Longair, M.S., \& Ryle, M., 1979, MNRAS, 188, 625.

[35] Longair, M.S., Ryle, M., \& Scheuer, P.A.G. 1973, MNRAS, 164, 243

[36] McCarthy, P. J., van Breguel, W., \& Kapahi, V.K., 1991, ApJ, 371, 478

[37] Mundell, C.G., Wrobel, J.M., Pedlar, A., \& Gallimore, J.F., 2003, ApJ, 583, 192

[38] Murgia, M., et al. 1999, A\&A, 345, 769

[39] Myers, S., \& Spangler, S., 1985, ApJ, 291, 52.

[40] Norman, M.L., 1996, in Energy Transport in Radio Galaxies and Quasars; eds. P.E. Hardee, A.H. Bridle, and J.A. Zensus, vol. 100 (ASP Conf. Ser. San Francisco), 319.

[41] O’Dea, C. P. 1998, PASP, 110, 493

[42] Onuchukwu, C.C., \& Ubachukwu, A.A., 2013, ApSS, DOI 10.1007/s10509-012-1325-x

[43] Orienti, M., \& Dallacasa, D., 2010, MNRAS, 406, 5290

[44] Parma, P., Murgia, M., Morganti, R., Capetti, A., de Ruiter, H. R., \& Fanti, R. 1999, A\&A, 344,7

[45] Pearson, T.J., \& Readhead, A.C.S., 1988, ApJ, 328, 114

[46] Perucho, M., \& Mart, J. M. 2002, ApJ, 568, 639

[47] Piner, B. G., Mahmud, M., Fey, A. L., \& Gospodinova, K. 2007, AJ, 133, 2357

[48] Polatidis, A.G., \& Conway, J. E., 2003, Proc. Astron.Soc.Aust., 20, 69

[49] Ryle M., \& Longair, M.S., 1967, MNRAS, 136, 123

[50] Ryś, S., 2000, A\&A, 355, 79

[51] Saikia, D.J., Jeyakumar, S.K., Wiita, P.J., Sanger, H.S., \& Spencer, R.E. 1995, MNRAS, 276,1215

[52] Scheuer, P.A.G., 1974, MNRAS, 166, 513.

[53] Scheuer, P.A.G., 1995, MNRAS, 277, 331.

[54] Schoenmakers, A. P., de Bruyn, A. G., Röttgering, H. J. A., \& van der Laan, H. 2000, MNRAS, 315, 395

[55] Stanghellini, C., Dallacasa, D., Venturi, T., An, T., and Hong, X. Y., 2009, Astronomiche Nachrichten, 330, 153

[56] Ubachukwu, A.A., 1998, Ap\&SS, 254, 23

[57] Wardle J.F.C., \& Aaron S.E., 1997, MNRAS, 286, 425

[58] Whitney A.R., 1971, Science, 173, 225 